\title{
APPLICATION DE LA THÉORIE DES NÉGOCIATIONS CONVERSATIONNELLES DE KERBRAT-ORECCHIONI ET DE LA NOTION DE STRATÉGIE À L'ANALYSE DES MESSAGES ASYNCHRONES DE L'INTERACTION VIRTUELLE
}

\begin{abstract}
A b s tra ct. Pirogowska Ewa, Applications de la théorie des négociations conversationnelles de Kerbrat-Orecchioni et de la notion de stratégie à l'analyse des messages asynchrones de l'interaction virttuelle. [Application of conversational theory of Kerbrat-Orecchioni and the notion of strategy in the analysis of asynchronous messages of virtual interaction]. Studia Romanica Posnaniensia, Adam Mickiewicz University Press, Poznań, vol. XXXVII/2: 2010, pp. 61-69. ISBN 978-83-232-2189-0. ISSN 0137-2475. DOI 10.2478/v10123-010-0015-1.
\end{abstract}

The written interactive register of internet discourse became the generous source for contemporary linguistics studies. This article tries to explain how to fit a modern conversational theory of C. KerbratOrecchioni to the analysis of interaction between humans in the computer asynchronous communication. The conclusions reached at intend to update notions like conversational strategy, conversational negotiation so as to apply them to the new communication means.

\section{PRÉLIMINAIRES - REGISTRE INTERACTIF ÉCRIT DE L'ÉCHANGE DISCURSIF DU FORUM DE DISCUSSION. MÉTHODOLOGIE APPLIQUÉE}

Les nouvelles formes de communication entre les humains, à savoir la conversation écrite dans le registre interactif via l'internet ou le réseau GSM, le système audio-tél, etc., ont remis en question les méthodologies traditionnelles utilisées pour examiner le fonctionnement de la langue. Plusieurs travaux, déjà élaborés dans ce domaine, laissent néanmoins place libre à des réflexions toutes neuves, issues à partir des études sur les corpus électroniques récemment apparus.

Nous avons entrepris une étude dans le champs des analyses conversationnelles qui ont pour point de départ la constatation que chaque échange communicationnel interactif (qui, par définition, a pour but d'échanger des messages) est une conversation.

Nous nous sommes précisément intéressée au registre interactif écrit des forums de discussion, dont l'interactivité est conditionnée par les délais de temps, c'est-à-dire que les messages sont répartis dans le temps. Le processus de réception/envoi n'est 
donc pas momentané, comme c'est le cas d'un bavardage sur la t'chatche. Le corpus lexical, base de nos analyses dont nous présentons aujourd'hui un court aperçu, est constitué d'un enregistrement authentique dont les références sont précisées dans la bibliographie. Les contributions ont été retranscrites sous format textuel Word.

En mettant sous examen l'échange dialogique du forum en question, nous l'avons traité comme un dialogue, spécifique et conditionné par son caractère du registre interactif écrit, mais quand même un dialogue entre plusieurs participants - une forme plurilogale en plein sens du mot.

\section{NÉGOCIATIONS CONVERSATIONNELLES. CAS SPÉCIFIQUE - L'ABANDON COMMUNICATIF}

Il nous semble judicieux d'aborder à l'ouverture de notre article la question des négociations conversationnelles que nous emploierons pour observer de plus près le déroulement de l'interaction enregistrée. Juste avant d'y passer, précisons que l'échange des messages de forum internet relève des organisations conversationnelles, voire dialogiques, vu les tours de parole qui ménagent les apports discursifs des participants. " Telle est [...] la spécificité du discours-en-interaction, d'être produit par plusieurs locuteurs qui prennent la parole "à tour de rôle" " (Kerbrat-Orecchioni, 2005 , p. 6). Cette alternance n'est pas pour autant une fin en soi, au moins dans le cadre des forums de discussion, mais un simple moyen au service de la construction $\mathrm{du}$ sens. Les analystes du flux conversationnel des tchacheurs remarquent, par contre, que c'est sur l'échange-même que l'intérêt des intervenants se concentre (p.ex. Dejond, 2002); effectivement, l'alternance des tours de parole sert dans ce dernier cas à maintenir un lien communicatif (pour ne pas trop dire «social», cf. K.-O., p. 6) ${ }^{1}$, l'échaffaudage d'un «texte» cohérent et cohésif étant une moindre préoccupation des sujets interactants. La fonction phatique du discours prévaut. Quant au forum, une certaine coopération, la co-construction du discours (K.-O., p. 20) des interactants conduit aux négociations conversationnelles.

Nous nous sommes appliquée à l'examen d'un ensemble des comportements mutuels qu'effectuent les contribuants des forums de discussion choisis, tout cela conformément aux remarques de Kerbrat-Orecchioni (K.-O., pp. 93 - 186). Vu les bornes spatiales de la publication actuelle, nous ne présenterons que quelques remarques et cela à l'appui des exemples provenant d'un seul forum de discussion. À titre d'illustration, nous présentons en ce lieu l'extrait de l'échange des posts (contributions) dont le fil conducteur est le chômage du mari de l'une des internautes intervenantes. Les énoncés ont été postés le 22 janvier 2009 entre 16:10:58 et 17:32:47. Pour que l'on parle de négociations, il faut qu'en leur fond il y ait un désaccord ou, au moins, un

\footnotetext{
${ }^{1}$ Pour les besoins de la lecture facile du présent article, nous avons raccourci le nom de Catherine Kerbrat-Orecchioni au sigle K.-O. vu les maints renvois à son œuvre récente (parue en 2005).
} 
manque d'accord. Les contributions ex_1, ex_2, ex_3 signalent qu'une querelle a lieu. Ex_4 constitue une sorte de commentaire ironique d'une troisième personne-négociatrice ; ex_5 et ex_7 constituent la défense de la soupçonnée coupable; l'explication des causes possibles du désaccord ; les posts ex_6, ex_8 et ex_9 sont les interventions des «négociatrices ».

Pour mettre de l'ordre dans nos délibérations, nous nous permettons en ce lieu de rassembler tous les points constituant une négociation interactionnelle, tels qu'ils sont définis par K.-O. : (1) l'existence des négociateurs ; (2) un objet à négocier ; (3) un état initial; (4) les procédures de négociation proprement dite; (5) un état final: quelle que soit l'issue. Suivant le schéma, le (1) peut être aligné à «chounette18 » et «Annabianca» qui entreprennent l'action négociatrice, ou, pour être précis, plutôt explicatrice. Le point (2) c'est l'éruption des émotions négatives ce qui n'est pas, selon les négociatrices, justifié. Les (3) (4) et (5) démontrent pleinement la spécificité de la réalité virtuelle puisque la connexion et la soutenance de l'interaction requiert moins d'effort de la part des sujets interactants et, ce qui s'ensuit, des grilles de l'analyse conversationnelle appliquées aux interactions en tête à tête. Est-ce qu'on peut vraiment parler d'un état initial clair et visible (lisible) si les posts se trouvent (sont émis et postés, alors publiés sur l'espace dialogal du forum) éloignés spatialement et temporellement ? «Spatialement» du point de vue graphique bien évidemment, en effet, nous n'avons recueilli que des contributions appartenant au fil discursif en question; en réalité, la discussion regardée dans sa totalité est fractionnée en plusieurs sous-fils qui s'entremêlent, se joignent et se disjoignent, et même sont menés séparément. Une telle confusion occasionne un ensemble interactionnel difficilement captivable. Sur ce point, on pourrait faire référence à une conversation sérieuse entre quelques participants qui sont interrompus incessamment par leur entourage, p.ex. par des enfants qui crient, par de nouveaux entrants, une station télé qui clignote (l'allusion aux publicités qui accompagnent le frame ${ }^{2}$ dialogal du forum dans sa version $\mathrm{http}$ ). L'autre marqueur, l'aspect de l'éloignement temporel, consiste en la répartition des messages conditionnée par le caractère asynchrone du forum de discussion. Même si les heures d'enregistrement des messages cités dans notre présentation ne sont pas très écartées entre elles, les contributions des participants ne se succèdent pas comme c'est le cas d'une interaction en temps réel ${ }^{3}$.

«Dans une interaction, les participants s'emploient à tenter de trouver un terrain d'entente leur permettant de réaliser cet "archi-but" commun qu'est la réussite de l'entreprise communicationnelle» (K.-O., p. 96). Ceci se passe très souvent, mais il arrive qu'une logique de négociation se transforme en celle d'un conflit avoué. Une

${ }^{2}$ Ici : le cadre graphique du site où la discussion est menée. En informatique, en général : (équivalent de trame en anglais) Entité indivisible destinée à être transmise par l'intermédiaire d'un réseau, elle contient à la fois les informations à transmettre et des champs de contrôle des données (source: http://dictionnaire.phpmyvisites.net/definition-FRAME-4531.htm )

${ }^{3}$ Il ne s'agit pas forcément de celle d'un tête à tête ; cela peut être aussi celle d'une conversation par téléphone ou même d'un bavardage sur la t'chatche au nombre limité de participants. 
interaction n'est jamais une harmonie prétablie, mais un compromis où toutefois les procédures collaboratives qui visent à résorber le désaccord parfois échouent. Il semble que, dans notre exemple, l'échec des négociations s'effectue par un abandon total; on dirait même un non-lieu de la résolution du problème communicatif.

Dans notre exemple, il n'y a aucune orientation vers un accord, au moins d'après le court extrait cité. K.-O. propose deux conditions nécessaires et suffisantes pour parler d'une négociation; la première concerne l'absence du désaccord (un ajustement immédiat). La seconde relève soit d'une dispute sans faire d'effort pour l'entente (et c'est le cas du fragment de l'échange observé), soit de la coexistence pacifique de deux lignes discursives divergentes. En observant le déroulement de l'échange dialogal asynchrone cité, on assiste à un déclenchement du conflit ouvert entre deux internautes (femmes). Une troisième et une quatrième personne y interviennent pour calmer l'ambiance de la dispute. Toutefois, chacune des deux parties campe sur ses positions dans une coexistence guerrière dont il n'y aura pas d'issue pacifique. Les opposantes sont restées sur des lignes différentes et conflictuelles. La suite de l'échange sur le forum cité fait voir que ce fil, parallèle au fil conducteur (chômage du mari de l'auteure), s'interrompe brusquement faute du désir d'une co-construction quelconque. En généralisant, dans les interactions virtuelles de tous types, un tel abandon de la collaboration communicationnelle a lieu assez souvent là où un conflit apparaît entre les internautes. Les négociations échouent donc à cause d'un non lieu, l'abandon d'une action communicationnelle en cours de la procédure négociatrice.

Les négociations conversationnelles ont la propriété « d'émerger de façon plus ou moins imprévisible dans une interaction qui n'a pas pour objectif préalable de traiter un désaccord précis » (K.-O., p. 99). La durée de la séquence négociative peut varier, il se peut que les sujets interactants s'entendent sur un épisode conversationnel ou, comme c'est le cas de notre exemple, interrompent soudainement l'échange en dépit des tentations explicatrices des autres intervenants. La clôture d'un des fils de l'interaction met un terme à toutes les tentatives d'alterner ou de transformer cette trame. Dans chaque négociation conversationnelle, les identités des participants et leur relation mutuelle sont l'objet de remaniements permanents et peuvent ne pas trouver d'issue positive.

\section{STRATÉGIE CONVERSATIONNELLE}

Dans les lignes qui suivent, nous allons centrer notre attention sur un terme proche des constatations sur la négociation conversationnelle, à savoir la stratégie conversationnelle. Dans l'analyse de discours, on observe divers emplois du terme en question et diverses définitions, selon les courants de recherche. La stratégie fait partie des conditions de production d'un discours (Bonnafous et Tournier, 1995), car, d'après nous, chaque sujet de l'interaction vise à obtenir un certain but communicationnel 
lors d'une rencontre, le dernier terme employé pour la substituer à n'importe quelle interaction, celle du monde virtuel incluse.

La structuration d'un acte de langage ${ }^{4}$ comporte deux espaces: « [...] un espace de contraintes qui comprend les données minimales auxquelles il faut satisfaire pour que l'acte de langage soit valide, [...] un espace de stratégies qui correspond aux possibles choix que les sujets peuvent faire de la mise en scène de l'acte de langage » (Charaudeau, 1995b). Les stratégies sont le fait d'un sujet, aussi bien individuel que collectif (comme c'est souvent le cas dans des forums de discussion professionnels) qui est conduit à choisir, de façon consciente ou non, un certain nombre d'opérations langagières. Dans le fragment de discussion que nous proposons, il serait probalement exagéré de parler d'une stratégie préalable, toutefois une sorte de stratégie naît inconciemment quand le conflit se déclenche. Les négociatrices, «chounette18» et «Annabianca», procèdent à minimaliser les émotions négatives en démontrant le mal-fondé de l'irritation de « mamancalinoux68» :

Le souci est que tu utilise ce post pour lui balancer je ne sais pas trop quoi alors qu'ici, elle n'a rien dit de particulier (Ex_9).

A plus forte raison, parler de stratégie n'a de sens que par rapport à un cadre de contraintes, qu'il s'agisse de règles, de normes ou de conventions. Il faut un but, une situation d'incertitude, une visée de résolution du problème. Les participantes de notre conversation de forum ne désirent pas que l'on interrompe le fil conducteur, c'est-àdire les conseils consolateurs portés auprès d'une internaute qui est dans une situation matérielle difficile à cause du chômage de son partenaire. Le comportement de «mamancalinoux68 » constitue sûrement une entrave à la règle (car il n'est pas gentil de se disputer s'il y a une personne qui a besoin d'aide psychologique). Dans les démarches communicationnelles des co-scripteurs, c'est-à-dire des participants de l'échange du forum (qui s'écrivent les uns aux autres = dialoguent à l'écrit) on assiste parfois à une sorte d'anti-stratégie conversationnelle (cf. Habrajska, 2002) parce qu'on peut observer plusieurs situations où un interlocuteur bloque les intentions communicatives impliquées par son adversaire, ou bien celui-là mène le fil conversationnel à un but opposé à celui qui a été envisagé par l'adversaire. Telle est la situation communicative puisée dans notre corpus où le contact non bona fide est engendré par le conflit issu d'émotions négatives. Son but ne peut pas être autre qu'une dégradation verbale du partenaire, et cela du simple fait que les règles de coopération ne sont pas suivies.

On emploie la notion de stratégie « par rapport à l'existence d'un cadre contractuel qui assure la stabilité et la prévisibilité des comportements de sorte que puisse intervenir un sujet qui aura à jouer soit avec les données du concret soit à l'intérieur de celles-ci » (Charaudeau, 1995a). Le développement total de la stratégie commu-

\footnotetext{
4 Pour rappeler : un acte de langage est un moyen mis en œuvre par un locuteur pour agir sur son environnement par ses mots : il cherche à informer, inciter, demander, convaincre, etc. son ou ses interlocuteurs par ce moyen. L'acte de langage désigne donc aussi l'objectif du locuteur au moment où il formule son propos. Cette idée a été développée par John Langshaw Austin (depuis 1962) et son étudiant John Searle.
} 
nicative dans l'échange des messages du forum cité nécessitera une étude infiniment plus ambitieuse et exhaustive que celle-ci ; nous avons uniquement voulu signaler l'optique de nos recherches (débutée par nos travaux : Pirogowska, 2005 et 2006) où la forme communicative nouvelle, l'échange asynchrone des messages, est un notable objet d'analyses linguistiques, surtout celles qui relèvent de l'analyse conversationnelle.

Pour dégager encore une dimension intéressante de l'échange asynchrone par rapport aux stratégies conversationnelles, jetons un coup d'œil sur le côté formel de l'enchaînement des contributions. La pratique de reprise de message précédent dans le message qui lui répond, ce qui est assez fréquent dans le forum analysé (considéré dans sa totalité), permet d'identifier plusieurs dimensions pertinentes. Les éléments que nous allons énumérer procèdent à l'organisation de la construction du sens global. Ils aident à l'élaboration du consensus ou, au contraire, perturbent la coopération. Tous les paramètres présentés ci-dessous relèvent de la notion de stratégie et l'organisent. Ainsi, les participants de l'échange emploient-ils :

a) des processus linguistiques comme l'anaphore, les procédés de thématisation ou les reformulations (p.ex. l'anaphore simple pronominale qui renvoit au mari de l'internaute-auteur du fil discursif :

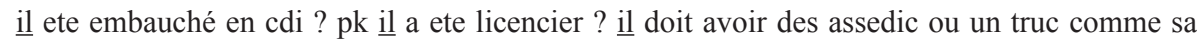
quand meme non?

(message de «julie1007» posté le 22 janvier 2009 à 15:35:06 dans le forum analysé)

b) des citations intégrales et/ou sélectives, des reprises bouleversant l'ordre séquentiel initial, p.ex.

A. ----julie---- a écrit :

B. Bon courage et bonne chance.

Toi tu faisais quel travail avant?

Tu as fais des études? Si j'ai bien compris tu peux pas reprendre le même boulot?

C. je suis adjointe de direction dans un hotel classe economique. mais le souci sont les horaires de travail je fini vers $21 \mathrm{~h}$ et deplus je fais des astreintes de nuit j'ai un BTS hotellerie restauration specialité gestion.

D. Message cité 2 fois

(où A. - c'est l'élément paratextuel (régi par le système du forum) qui précise l'auteur du message ; B. - le message précédent auquel on repond ; C. - la réponse ; D. - l'élément paratextuel (régi par le système du forum) qui précise le nombre de citations). Message de petitemaman 80 posté le 22 janvier 2009 à 15:42:05.

c) des commentaires exclamatifs, adressés à l'interlocuteur ou au public, de forte tension affective ; ces commentaires sont souvent métadiscursifs (effet de loupe, p.ex. [...] sur ta façon de parler un peu brut, Ex_1);

d) des co-constructions (p.ex. les structures bipartites « si..., par contre... », cf. Sacks, 1992, collaboratively built sentences). 


\section{REMARQUES FINALES}

L'échange des messages asynchrones crée une situation énonciative semblable à l'interaction en face à face, donc au dialogue oral. N'oublions pas toutefois qu'une conversation c'est l'interaction pure dont le critère fondamental est la co-présence des interlocuteurs. Ce n'est pas le cas, d'après la définition-même, de l'échange asynchrone. « Là, on a affaire à une situation énonciative différente de la conversation quotidienne: la réponse de l'interlocuteur à un message est différée dans le temps. La notion d'échange asynchrone renvoie cependant à celle de dialogue, qui, en restant une sorte d'interaction, n'est pas si strictement limité par l'encadrement temporel » (Pirogowska, 2005, p. 121). Soulignons néanmoins que l'un des synonymes du mot dialogue est la négociation qui est indispensable pour aboutir à la conclusion d'un accord.

La chaine des contributions de l'espace dialogal asynchrone est basée sur le principe de l'implicativité séquentielle (sequential implicativness, Schegloff, Sacks, 1973, pp. 289-327, cités aussi par Mondada, 1999). Les relations qui s'établissent dans la temporalité de l'alternance de la parole d'un locuteur à un autre sont en effet constructives pour l'organisation de chaque dialogue, y compris l'interaction asynchrone. L'interlocuteur, dans son intervention négociatrice, complète le message initial d'un nouveau contenu sémantique mais procède aussi à la création des attentes et contraintes normatives. Il existe donc un certain contrôle de l'espace intersubjectif qui est de facto le contrôle de la compréhension publique.

\section{L'EXTRAIT DU CORPUS LEXICAL}

Source : Forum de discussion "Famille" du site www.doctissimo.fr, fil du 22 janvier 2009 (les énoncés postés entre 16:10:58 et 17:32:47) sous le titre Je suis en panique .... mon mari vient de perdre son travail, consulté le 20 juin et le 24 septembre 2009. L'ortographe authentique.

Ex_1

Auteur : «mamancalinoux 68 », heure exacte non enregistrée; commentaire concernant la présence de l'internaute «titite 63 » avec qui «mamancalinoux 68 » est en désaccord

ah $\oplus_{c ̧}$ fait aucun doute sur ta façon de parler un peu brut,dès que tu vois «mamancalinoux» en pseudo $\odot \cdot \odot \cdot \odot \cdot \odot$

Ex_2

Début de la querelle, auteur: «titite63», en réponse à Ex_1

Mais n'importe quoi hein !! T'es vraiment dans ton délire.-: 
Ex_3

Réponse de «mamancalinoux 68 »

tu te définis bien

t'es signalé:; , moraliste et tu en sors,laisse moi rire !

Ex_4

«chounette 18 », en réponse à Ex_3

sympa de poluer le poste de qqun qui a besoin !!!!

\section{Ex_5}

«mamancalinoux68», en réponse à Ex_4

nan tu fais erreur de la personne

donne des dons après on verras,tiens petitefé est dans le besoin aussi de plus jette un coup

d'œil au poste,et elle est très sérieuse et bien dans le besoin

Ex_6

« chounette 18 », en réponse à Ex_5

excuse mais c'est toi qui vens d'attaquer titite !!! :

\section{Ex 7}

«mamancalinoux68», en réponse à Ex_6

elle m'a traité de conne désolé mais j'ai vu la miss sur d'autre discutions et c pas une tendre,ni une nana que $\mathrm{j}$ envie de connaitre!

\section{Ex 8}

«chounette 18 », en réponse à Ex_5

mais tu l'a attaquée combien de fois pour qu'elle te dise ça?

pi que tu l'est vue sur d'autres discutions j'en est rien a battre !!

Ex_9

«Annabianca», en réponse à Ex_7

Le souci est que tu utilise ce post pour lui balancer je ne sais pas trop quoi alors qu'ici, elle n'a rien dit de particulier.

\section{BIBLIOGRAPHIE}

Bonnafous, S., \& Tournier, M. (1995). Analyse du discours, lexicométrie, communication et politique. Langages 117, 67-81.

Charaudeau, P. (1995a). Le dialogue dans un modèle de discours. Cahiers de linguistique française, 17, 141-178.

Charaudeau, P. (1995b). Une analyse sémiolinguistique du discours. Langages 117, 96-111. 
Dejond, A. (2002).La cyber@langue française. Tournai : La Renaissance du Livre.

Habrajska, G. (2002). Strategie konwersacyjne w internetowych grupach dyskusyjnych. Język a komunikacja, 4, (Jezzyk trzeciego tysiaclecia), 161-174.

Kerbrat-Orecchioni, C. (2005). Le discours en interaction. Paris : Armand Colin.

Mondada, L. (1999). Formes de séquentialité dans les courriels et les forums de discussion. Récupéré sur http://alsic.u-strasbg.fr

Pirogowska, E. (2006). L'oralité dans la communication asynchrone via Internet sur les exemples du discours du cyberespace français et italien. Studia Romanica Posnaniensia, 33, 169-185.

Pirogowska, E. (2005). L'oralité secondaire dans le discours du cyberespace français et italien, thèse de doctorat. Poznań : Université Adam Mickiewicz.

Sacks, H. (1992). Lectures on Conversation. Oxford : Basil Blackwell.

Schegloff, E., \& Sacks, H. (1973). Opening up closing. Semiotica, 8, 289-327. 